\title{
Modelagem tridimensional de objetos conceituais de matemática
}

\author{
Delair Bavaresco \\ Docente. Instituto Federal de Educação, Ciência e Tecnologia do Rio Grande do Sul \\ (IFRS - Campus Bento Gonçalves) \\ (delabavaresco@hotmail.com) \\ Pétra Thalía De Toni \\ Instituto Federal de Educação, Ciência e Tecnologia do Rio Grande do Sul \\ (IFRS - Campus Bento Gonçalves) \\ (petratoni157@gmail.com) \\ Rafael Jonatan Pertile \\ Instituto Federal de Educação, Ciência e Tecnologia do Rio Grande do Sul \\ (IFRS -Campus Bento Gonçalves) \\ (rafapertile41@gmail.com) \\ Vicente Kinalski Junior \\ Instituto Federal de Educação, Ciência e Tecnologia do Rio Grande do Sul \\ (IFRS -Campus Bento Gonçalves) \\ (vicente_kinalski@hotmail.com)
}

\begin{abstract}
Resumo: Este trabalho apresenta resultados de um processo investigatório voltado para a modelagem tridimensional de objetos conceituais de matemática e confecção por meio de uma impressora 3D. Imersa na atmosfera da quarta revolução industrial, a qual tem no uso de impressoras 3D uma de suas tendências, a pesquisa que resultou na produção desse trabalho ancora-se nos pressupostos teórico metodológicos de Pesquisa de Desenvolvimento em Projetos Experimentais e na ideia de aprendizagem significativa. Os objetivos centram-se na resolução de dois problemas: a modelagem e confecção de uma peça composta por três cilindros ortogonais e a definição de uma metodologia para confecção de superfícies quádrica com espessura uniforme de paredes. Isso tudo com aplicação de conceitos e conhecimentos matemáticos para o dimensionamento e posicionamento preciso. O desenvolvimento e os resultados obtidos mostram o processo de resolução dos problemas e a obtenção de modelos tridimensionais que atendem aos objetivos da pesquisa, bem como de peças com boa definição, bom acabamento e de acordo com os conceitos matemáticos explorados, mostrando que o conhecimento matemático constitui-se como base fundamental para a modelagem tridimensional precisa e de qualidade.
\end{abstract}

Palavras-chave: Impressora 3D; Superfícies quádricas; Aprendizagem Significativa.

\section{Three-dimensional modeling of conceptual math objects}

\begin{abstract}
This paper presents the results of a research process aimed at the three - dimensional modeling of conceptual objects of mathematics and confection through a 3D printer. Immersed in the atmosphere of the fourth industrial revolution, which has in the use of 3D printers one of its tendencies, the research that resulted in the production of this work is anchored in the theoretical methodological assumptions of Development Research in Experimental Projects and in the idea of meaningful learning. The objectives are to solve two problems: the modeling and preparation of a piece composed of three orthogonal cylinders and the definition of a methodology for the preparation of quadratic surfaces with uniform wall thickness. All these are with application of concepts and mathematical knowledge for the sizing and precise positioning. The development and results obtained show the process of solving problems and obtaining three-dimensional models that attend the objectives of the research, as well as pieces with good definition, good finishing and according to the mathematical concepts explored,
\end{abstract}


showing that mathematical knowledge it constitutes a fundamental basis for precise and quality threedimensional modeling.

Keywords: 3D Printer; Quadratic surfaces; Meaningful Learning.

\section{INTRODUÇÃO}

Vivemos numa era de crescentes mudanças tecnológicas, as quais implicam em mudanças sociais e passam a nortear nossa forma de vida. Schwab (2016), autor do livro intitulado A Quarta Revolução Industrial, afirma que:

Estamos no início de uma revolução que alterará profundamente a maneira como vivemos, trabalhamos e nos relacionamos. Em sua escala, escopo e complexidade, a quarta revolução industrial é algo que considero diferente de tudo aquilo que já foi experimentado pela humanidade (p. 11)

De acordo com o autor, esse momento, também chamado de Revolução 4.0, acontece após três processos históricos transformadores. $O$ primeiro foi à mecanização da produção entre 1760 e 1830 . Posteriormente, por volta de 1850, a eletricidade permitiu a manufatura em massa. O terceiro marca a chegada da eletrônica, da tecnologia da informação e das telecomunicações, já no século XX.

No Fórum Mundial de Davos, em janeiro de 2016, houve uma sintetização das principais ideias acadêmicas relacionadas à Revolução 4.0: nanotecnologias, neurotecnologias, robôs, inteligência artificial, biotecnologia, sistemas de armazenamento de energia, drones e impressoras 3D.

As impressoras 3D já ocupam um papel de peso quando o assunto é a Quarta Revolução Industrial, afirma Vinícius Miguél, um dos diretores da CNC Brasil, em entrevista para a revista Exame. Par ele:

A Revolução Industrial não vai acontecer, ela já está acontecendo. A
tendência agora é que ela ganhe ainda mais força e notoriedade. Eu
não tenho dúvidas de que os feitos já obtidos, em várias áreas, a partir
do uso da Impressora 3D tenham sido, e continuem sendo, bastante
significativos. O que me faz concluir que esta tecnologia já é uma das
grandes impulsionadoras da Revoluçãa Industrial (DINO, 2016).

Essa atmosfera, pós-revolução digital, tem inspirado aquilo que alguns autores denominam de Cultura Maker, a qual tem como uma de suas máximas a expressão 
do filósofo Aristóteles: É fazendo que se aprende a fazer aquilo que se deve aprender a fazer. Nesse processo, a prototipagem rápida e a fabricação digital têm ganhado espaço com a popularização das impressoras 3D, as quais vêm invadindo muitos ambientes escolares nos últimos anos.

Inevitavelmente, a Revolução 4.0 gera significativas implicações no sistema educacional escolarizado, é o que afirma Sanchez (2017).

Imaginem vocês que $65 \%$ das crianças que entrarem hoje na escola primária irão trabalhar em empregos que hoje não existem! É urgente uma ação adaptativa de todos e, principalmente, das escolas! [...] Atualmente, temos um ambiente corporativo - Workplace 3.0 - onde se verifica o prazer no aprendizado, a grife perdeu espaço para os conceitos, alta relevância no significado das tarefas e aprendizado baseado em projetos. Fazendo um paralelo com a nossa escola, concluímos que estamos ainda na versão 2.0. Temos um modelo educacional analógico para uma geração digital, por isto não conseguimos colher bons resultados, pois o serviço prestado não é absorvido pelo aluno (2017, p. 01).

É nessa atmosfera que se desenvolveu o presente trabalho, o qual apresenta resultados de um processo investigativo resultante de um projeto de aprendizagem de modelagem tridimensional. O referido projeto teve como propósito a confecção, por meio de uma impressora 3D, de objetos conceituais de matemática, explorando conceitos de dimensionamento preciso para obtenção de objetos bem definidos e de boa qualidade de impressão.

Conforme Sanchez (2017), este estudo insere-se numa perspectiva contemporânea em que a ciência e a tecnologia assumem lugar de destaque na conjuntura socioeconômica globalizada. Nessa perspectiva, a Matemática configurase como um dos alicerces desse processo, por meio de estratégias de resolução de problemas, modelagem matemática e simulação numérica e, ainda, técnicas de prototipagens e seus processos.

Ancorada nas discussões contemporâneas de desenvolvimento científico e tecnológico a partir da educação e com vistas à inovação, a metodologia de Projetos Experimentais utilizada possui, como uma de suas características, o aprofundamento da compreensão do fenômeno sob investigação pelo pesquisador enquanto a atividade está em andamento.

O projeto de aprendizagem de modelagem tridimensional, que está voltado para prototipagem de elementos geométricos, explorando conceitos matemáticos, 
gerou dois problemas centrais descritos a seguir, os quais levaram a amplas discussões e a resultados significativos.

O primeiro problema em estudo surgiu da necessidade de se confeccionar uma origem do sistema cartesiano tridimensional com uso de uma impressora 3D e pode ser descrito da seguinte forma: três cilindros de $2 \mathrm{~cm}$ de diâmetro e $8 \mathrm{~cm}$ de altura se conectam ao meio formando uma peça única. Essa peça é depositada em um plano de modo que os três cilindros tocam o plano conforme ilustra a Figura 1a. Para a confecção desse cilindro por meio de uma impressora 3D, foi necessário gerar um prolongamento, em forma de cunha cilíndrica, na extremidade de cada um dos cilindros, de modo a resultar numa superfície plana de sustentação e aderência ao plano de impressão, conforme ilustra a Figura $1 \mathrm{~b}$.

Figura 1: Representação do problema de estudo
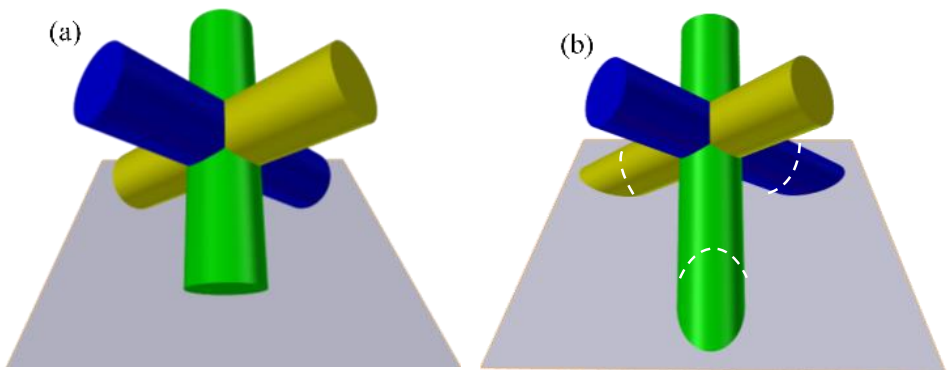

Fonte: Desenvolvido pelo Autor.

O segundo problema consistiu em elaborar uma metodologia de modelagem tridimensional de superfícies quádricas, definidas por funções de duas variáveis, para posterior confecção por meio de uma impressora 3D. O grande desafio consistiu em superar a dificuldade de se obter uma superfície cujos pontos satisfizessem funções quadráticas de duas variáveis e apresentassem uma uniformidade da espessura da peça em todos os pontos. O processo de resolução desses problemas e a produção dos objetos pretendidos são os principais resultados deste trabalho.

\section{FUNDAMENTAÇÃO TEÓRICA}

A pesquisa que motivou a escrita deste trabalho baseia-se em algumas propostas teórico-metodológicas que se adequam ao desenvolvimento de pesquisas em ambientes educacionais. Uma proposta que vem crescendo no interesse de pesquisadores/autores é a de Pesquisa de Desenvolvimento em Projetos 
Experimentais como uma tradução de Design Experiments in Educational Research, introduzida, em 1992, por Brown e Collins (COLLINS, et. al, 2004).

Essa perspectiva vem se apresentando como uma proposta de inovação educacional, sobretudo para o ensino e a aprendizagem de ciências exatas, como é o caso da Física e da Matemática. Nessa proposta, em vez de serem estimulados por aulas tradicionais, os estudantes devem buscar respostas a questões complexas, muitas vezes multidisciplinares, e devem apresentar um produto final como resultado de suas pesquisas.

Para Cobb (2003), essa perspectiva enfatiza o aprofundamento da compreensão do fenômeno sob investigação pelo pesquisador enquanto a atividade está em andamento, de modo a aplicar diferentes conhecimentos. Em geral, a pesquisa de desenvolvimento é um processo interativo, intervencionista e orientado com base em fundamentos teóricos, com vistas à prática educacional. Na pesquisa de desenvolvimento, os projetos possuem uma abordagem pragmática dupla "- formas particulares de aprendizagem - e uma orientação teórica - desenvolvendo teorias específicas de domínio ao estudar sistematicamente essas formas de aprendizagem e os meios de apoiá-las" (COBB, 2003, p. 09).

Para Doerr e Wood (2006), essa metodologia segue dois princípios norteadores: o desenvolvimento de um processo ou de um produto, e os vários ciclos de análise necessários, com a finalidade de aprimorar o produto. No caso deste trabalho, o produto final, mesmo tendo um propósito bem definido, coloca-se em segundo plano. Em se tratando de um ambiente educacional, o processo torna-se a principal ênfase, pois é nele que o aprendizado se torna mais significativo por meio da aplicação dos conceitos teóricos previamente estudados.

Desse modo, a pesquisa filia-se, também, na proposta metodológica de Aprendizagem Significativa (AUSUBEL, 1965). Para o autor, a Aprendizagem Significativa "é o mecanismo humano, por excelência, para adquirir e armazenar a vasta quantidade de ideias e informações representadas em qualquer campo de conhecimento" (AUSUBEL 1965, p. 58). Para que o mecanismo seja acionado, é preciso que o aprendiz já possua algum conhecimento prévio, ou seja, já deve existir uma estrutura cognitiva em funcionamento.

Para Pelizzari et al. (2002), a aprendizagem significativa vem a ocorrer quando: 
os indivíduos apresentam uma organização cognitiva interna baseada em conhecimentos de caráter conceitual, sendo que a sua complexidade depende muito mais das relações que esses conceitos estabelecem em si que do número de conceitos presentes (p.38).

Ou seja, ocorre a aprendizagem significativa quando criamos uma rede de relações daquilo que aprendemos, organizando tais conhecimentos em uma ordem cronológica ligada a uma hierarquia, que provém de nossa capacidade de compreender e relacionar aquilo que aprendemos.

A Pesquisa de Desenvolvimento em Projetos Experimentais, de acordo com Barbosa e Oliveira (2015), tem no centro do processo investigativo o delineamento, elaboração do artefato em sua primeira versão. É o que se pode denominar de prototipagem, sendo que o desenvolvimento refere-se ao processo contínuo de aperfeiçoamento por meio da avaliação sistemática.

Segundo Vaz (2004, p. 36), no design experiments "o enfoque está nos significados construídos pelos aprendizes, tendo como objetivo principal analisar o seu pensamento matemático, ou seja, os processos pelos quais esses pensamentos se constroem e suas modificações". Sztajn et al. (2013) destacam o uso dessa metodologia como um campo emergente para as ciências da aprendizagem e desenvolvimento profissional, ligado às questões de desenvolvimento tecnológico com base na ciência aplicada.

\section{PROCEDIMENTOS METOLÓGICOS}

O conhecimento científico tem sua origem, de modo mais formal e organizado, com Galileu Galilei (1564-1642); no entanto, a racionalização do conhecimento, numa distinção com conhecimento mítico, tem origem na Grécia Antiga, em meados do século VII a.C. O conhecimento científico se dá pela determinação de um fenômeno ou de um objeto de investigação e da definição de um método para a realização dessa investigação.

A Ciência pode ser definida como um conjunto de saberes produzidos de forma racional, metodológica, associada à observação, identificação, descrição, investigação experimental e explanação teórica de fenômenos. É um conhecimento objetivo, metódico, passível de demonstração e comprovação (FONSECA, 2002). 
Com relação ao método, Tartuce (2006, p. 12) defende que "os métodos científicos são as formas mais seguras inventadas pelos homens para controlar 0 movimento das coisas que cerceiam um fato e montar formas de compreensão adequada dos fenômenos".

Quanto à caracterização, a pesquisa que deu origem à escrita deste texto pode ser definida como pesquisa aplicada, uma vez que seus objetivos são voltados para a resolução de problemas. Para Barros e Lehfeld (2000, p. 78), a pesquisa aplicada tem como objetivo "contribuir para fins práticos, visando à solução mais ou menos imediata do problema encontrado na realidade”. Appolinário (2004, p. 152) destaca que pesquisas aplicadas têm o objetivo de "resolver problemas ou necessidades concretas e imediatas".

É nesse viés de resolução de problemas aplicados que a pesquisa se insere. Nesse caso, a modelagem tridimensional tem como propósito a confecção, por meio de uma impressora 3D, de objetos com exploração de elementos geométricos, com atenção maior ao processo de aprendizagem, que é proporcionada pelo processo de investigação.

Atualmente, existem três tipos de impressora 3D disponíveis no mercado. Estereolitografia, Impressão direta por Luz e Extrusão. A Estereolitografia é um processo com um laser e um tipo de resina líquida. A Impressão direta por Luz (DLP) é um processo similar à Estereolitografia, porém, nesse modelo, o equipamento usa uma fonte de luz diferente do laser. O sistema por extrusão é o método mais comum e presente nas máquinas mais baratas. O processo funciona a partir de um extrusor que libera camadas subsequentes de um material plástico aquecido. Seguindo as orientações do arquivo de impressão, a cabeça extrusora libera o material de camada em camada, dando assim os contornos para o objeto desejado (GARRETT, 2016).

Os testes realizados pelos autores deste trabalho deram-se com uso de uma impressora que opera pelo processo de extrusão, uma impressora da marca Cliever, modelo Cliever CL1 - Black Edition. As limitações do processo de extrusão são apontadas pela baixa resolução do material impresso e pelo grande tempo para a confecção de formas geométricas mais complexas (GARRETT, 2016). No entanto, outra limitação pôde ser observada durante os testes: ocorre que, para a confecção de peças que possuem regiões horizontais e que não ficam em contato com a base de impressão, é necessária a construção de material de suporte para que o filamento possa ser depositado. Esse processo resulta em aumento do tempo de impressão, 
maior consumo de material e piora a resolução dos acabamentos, uma vez que o material de suporte precisa ser removido após a impressão.

Algumas estratégias de modelagem e posicionamento de peças podem ser usadas para contornar o problema do material de suporte. É o caso da peça formada pela união ortogonal dos três cilindros descrita anteriormente. Dessa dificuldade, surgiu a ideia de posicionar os três cilindros, todos inclinados para que a peça pudesse ser confeccionada sem construção do material de suporte.

A modelagem tridimensional para impressão 3D, para o tipo de impressora utilizada, pode ser realizada em qualquer software que permita a exportação para o formato reconhecido pelo sistema operacional da impressora. Algumas opções como AutoCAD, Solidworks e similares possuem alto potencial de confecção de modelos tridimensionais para essa finalidade. No entanto, são opções comerciais com alto custo de licenças de instalação. Algumas alternativas como Tinkercad, Sketchup, Blender, entre outros, são opções de utilitários com licença livre e que permitem a modelagem com riqueza de detalhes.

Nossa opção foi pelo uso do Blender, por ser um software de modelagem 3D bastante versátil e um dos mais populares do mercado, além de oferecer uma enorme variedade de ferramentas, dando aos seus usuários uma liberdade completa em termos de design. Além disso, conta com a comunidade mais ativa e documentação mais extensa de todos, inclusive com um portal brasileiro para sanar a maioria das dúvidas que possam surgir.

O processo de modelagem tridimensional com o Blender parte da utilização e transformação de objetos geométricos predefinidos. Dentre os principais, destacamse plano, cubo, cilindro, cone, esfera e objeto toroidal. As principais estratégias de transformação desses objetos dão-se por meio da elevação e extrusão de vértices e arestas, bem como do uso das operações booleanas de diferença, união e intersecção de objetos.

O potencial para a modelagem de objetos com exploração de elementos geométricos, que o software possibilita, dá-se pela possibilidade de dimensionamento, tanto para as características primárias dos objetos, quanto para posicionamento e operações boolianas. As ferramentas de localização e rotação de objetos dão flexibilidade para um posicionamento preciso a partir de valores inseridos via teclado pelo usuário. A Figura $2 a$ mostra as operações de elevação em uma unidade e de extrusão horizontal em $50 \%$ das arestas e vértices da parte superior de um cubo de 
dimensão inicial igual a 2. As Figuras $2 b$ e 2c ilustram a operação de subtração de dois cilindros de diferentes raios e alturas, permitindo a obtenção de um cilindro oco de espessura igual a $2 \mathrm{~mm}$.

Com uma sequência de várias operações é possível modelar formas complexas de acordo com a necessidade e os objetivos do usuário. O grande desafio, em muitos casos, é a determinação dos parâmetros que regem essas operações, como é o caso dos dois problemas que seguem.

Figura 2: Operações elementares com objetos no software Blender.
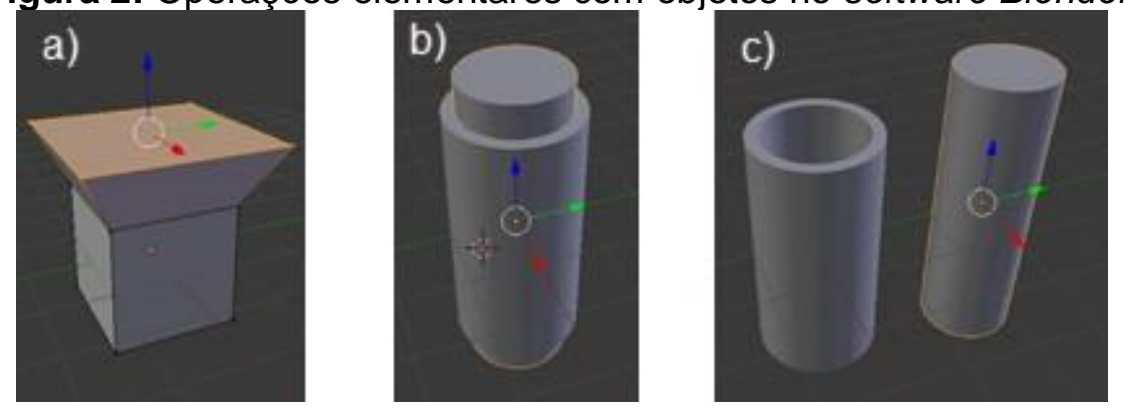

Fonte: Desenvolvido pelo Autor.

\section{RESOLUÇÕES DOS PROBLEMAS}

\subsection{O problema dos três cilindros}

O processo de impressão tridimensional demanda a modelagem de objetos de tal modo que estes possuam uma base de apoio e fixação para que as sucessivas camadas de filamento possam ser depositas. Diante disso, o primeiro desafio para obtenção de uma peça regular foi a determinação do ângulo que cada cilindro faz com relação ao plano horizontal. Esse resultado foi alcançado ao comparar a posição da peça com um plano inclinado que corta os três eixos cartesianos a uma mesma distância da origem, conforme ilustra a Figura 3a. Planos com essa característica tem equações do tipo $x+y+z=k$ (nesse caso, escolhemos $k=\mathbf{3}$ ) conforme ilustra a Figura 3b, traçada no software Maple. A partir disso, podemos obter a inclinação do plano com relação a cada um dos eixos (que correspondem aos cilindros) com base no ângulo formado pelo vetor diretor do plano e o vetor diretor de cada um dos eixos coordenados, conforme mostra a Figura 3c. Em vista disso, obtém-se a inclinação dos 
cilindros a partir do ângulo formado pelos vetores $n^{\rightarrow}=(1,1,1)$, que é o vetor diretor do plano de equação $x+y+z=\mathbf{3}$, $\mathrm{e}^{x^{\rightarrow}}=(1,0,0)$, que é o vetor diretor do eixo $\mathrm{x}$, por meio da relação:

$$
\cos \alpha=\frac{\vec{n} \cdot \vec{x}}{|\vec{n}| \cdot|\vec{x}|} \Rightarrow \cos \alpha=\frac{\sqrt{3}}{3} \quad \Rightarrow \quad \alpha=54,73560
$$

Figura 3: Comparação da alocação da peça com o plano dado pela equação.

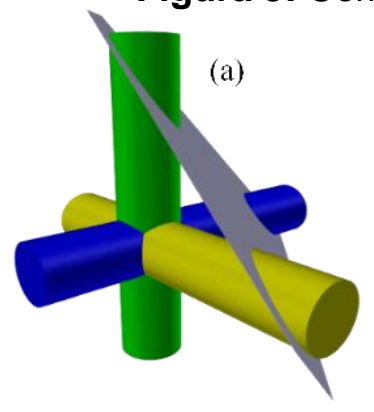

(b)

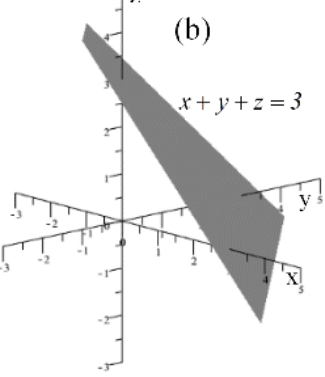

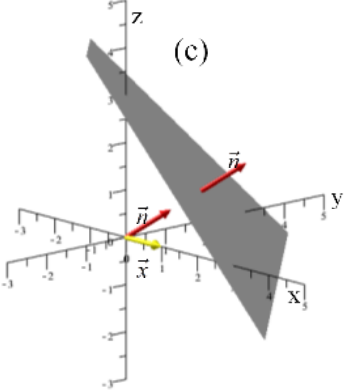

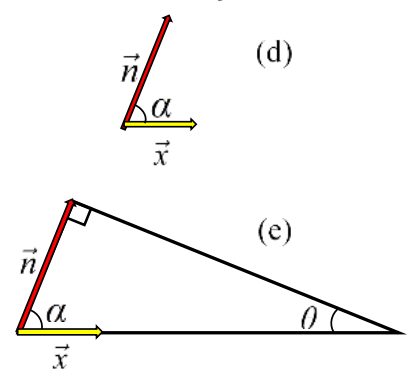

Fonte: Desenvolvido pelo Autor.

A Figura 3d mostra essa composição. A Figura 3e ilustra a obtenção de um triângulo formado por segmentos de retas $s_{1}$ e $s_{2}$ suportadas pelos vetores $\vec{n} \mathrm{e} \vec{x} \mathrm{e}$ pelo segmento contido no plano e que intersecta $s_{\mathbf{1}}$ e $s_{\mathbf{2}}$. Pela condição de ortogonalidade do vetor diretor do plano, o triângulo é retângulo, permitindo a obtenção do ângulo $\theta$ procurado, dado por:

$$
\theta=90^{\circ}-\alpha \Rightarrow \theta=35,2646^{\circ}
$$

O resultado dados pela equação (1) permitem a obtenção das demais razões trigonométricas para o ângulo $\alpha$ por meio de relações fundamentais da trigonometria,

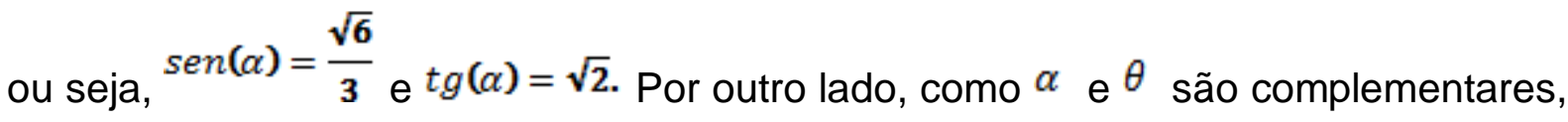
$\operatorname{sen}(\alpha)=\cos (\theta) \mathrm{e}^{\cos (\alpha)=\operatorname{sen}(\theta)}$, obtém-se $\operatorname{sen}(\theta)=\frac{\sqrt{3}}{3}, \cos (\theta)=\frac{\sqrt{6}}{3} e^{\operatorname{tg}(\theta)=\frac{\sqrt{2}}{2}}$.

Na sequência, o segundo desafio foi obter a medida do comprimento da cunha que deve ser somada aos oito centímetros do cilindro definido inicialmente, conforme ilustra a Figura 4a. Considerando-se a inclinação $\theta$ obtida e o diâmetro de $2 \mathrm{~cm}$ do cilindro, definido inicialmente, é possível construir o triângulo retângulo mostrado na 
Figura 4b. A partir da razão $\operatorname{tg}(\theta)$ desse triângulo, obtemos a medida $l$ procurada dada por:

$$
\operatorname{tg}(\theta)=\frac{2}{l} \Rightarrow \frac{\sqrt{2}}{2}=\frac{2}{l} \Rightarrow l=2 \sqrt{2}
$$

Por fim, para obtermos o posicionamento exato da peça, de modo a possibilitar a confecção por meio da impressora 3D, precisamos determinar a altura do centro da peça com relação à horizontal. Esse processo se faz necessário, pois o software Blender toma como referência o seu centro geométrico para posicionamento da peça que, neste caso, coincide com o centro de cada um dos cilindros considerando o comprimento total $8+2 \sqrt{2} \mathrm{~cm}$.

Figura 4: Esquema para determinação do tamanho da cunha e da altura da peça.

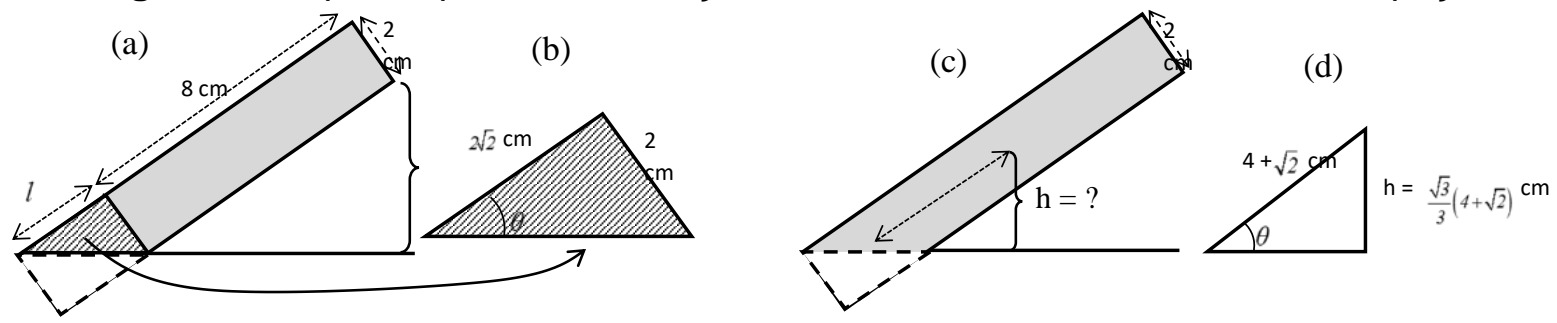

Fonte: Desenvolvido pelo Autor.

A Figura 4c mostra a obtenção de um triângulo retângulo obtido pela projeção vertical do centro dos cilindros até o plano suporte e a projeção do centro até o plano formando um ângulo $\theta$. Da aplicação da razão $\sin (\theta)$, tomada no triângulo mostrado na Figura 4d, obtém-se a altura procurada que é dada por:

$$
\sin (\theta)=\frac{h}{4+\sqrt{2}} \Rightarrow h=\frac{\sqrt{3}}{3}(4+\sqrt{2})
$$

\subsection{O problema das superfícies quádricas}

Como já mencionado nos procedimentos metodológicos, a modelagem tridimensional com o uso do software Blender consiste na transformação de objetos pré-definidos por meio de operações elementares, tais como extrusão e elevação de 
vértices e arestas, entre outras. Além disso, as operações booleanas permitem a realização de operações de diferença, união e intersecção de sólidos.

Desse modo, os primeiros protótipos de superfícies quádricas foram obtidos por meio da modelagem de um sólido limitado externamente pela superfície quádrica que satisfaz uma determinada equação quadrática em três variáveis. Posteriormente, foi modelado outro sólido com dimensões menores, de tal modo que a diferença entre um e outro resultava na superfície desejada, com determinada espessura préestabelecida.

Tratando-se de superfícies curvas, oriundas de equações não lineares, os primeiros modelos resultaram na produção de peças com espessuras não homogêneas, ocasionando regiões frágeis, como ilustra a Figura 5. Além disso, essa metodologia mostrou-se limitada, permitindo gerar apenas algumas superfícies, pois superfícies quádricas, tais como um hiperboloide elíptico de uma folha, dado pela Equação (5), não respondem uniformemente às operações de extrusão horizontal e de elevação, por exemplo, pois possuem coeficientes distintos para cada variável.

$$
\frac{4 x^{2}}{25}+\frac{4 y^{2}}{9}-\frac{3 z^{2}}{25}=1
$$

Figura 5: Peças de baixa qualidade, pouca definição e irregularidades de espessura.

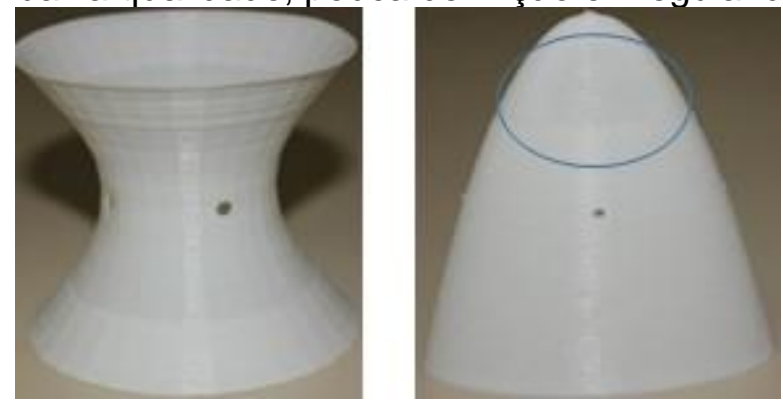

Fonte: Desenvolvido pelo Autor.

A estratégia utilizada para modelar e confeccionar qualquer superfície culminou na modelagem de superfícies a partir da união de sucessivos planos, de dimensões cada vez menores, localizados em pontos do espaço que satisfazem funções de duas variáveis, conforme ilustra a Figura 6. Nesse caso, é mostrada a obtenção do paraboloide hiperbólico dado pela equação:

$$
z=\underline{(\Theta)^{2}-y^{2}}
$$

com 16, 64 e 4.096 planos respectivamente. Nesse processo, qư⿱ánto menores forem esses planos, melhor será a suavidade da superfície gerada, ou seja, as dimensões de cada plano tendem a zero e a quantidade de planos tende ao infinito. 
Outro ponto de estudos, e, talvez, o mais desafiador, foi o problema da uniformidade da espessura em todos os pontos da peça. Para a obtenção de peças com espessuras uniformes, fez-se necessária a realização da operação de extrusão, considerando-se a não linearidade das superfícies. Desse modo, para cada ponto da superfície fez-se necessário determinar uma direção de extrusão, a direção do vetor normal à superfície naquele ponto. O vetor normal a uma superfície é o vetor diretor do plano tangente naquele ponto.

Figura 6: Representação das etapas de modelagem das superfícies.

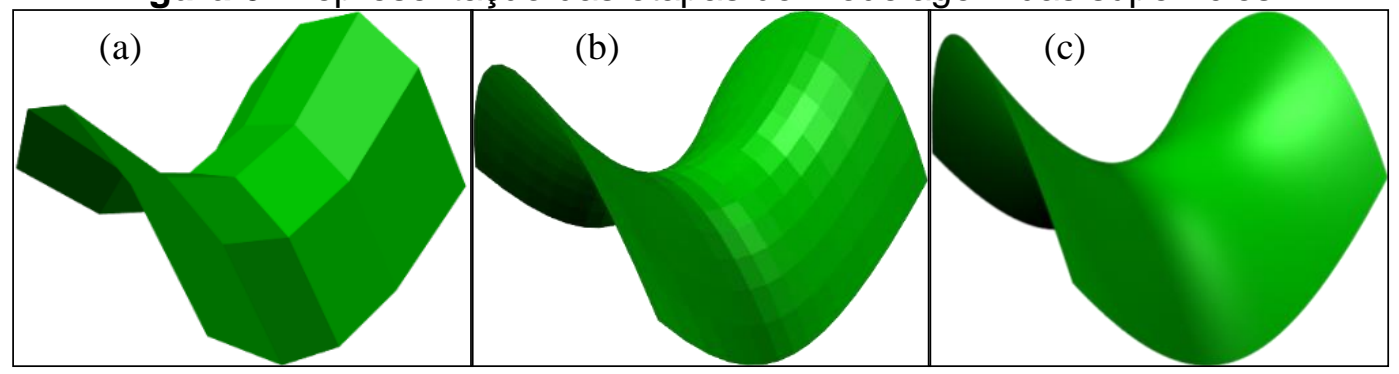

Fonte: Desenvolvido pelo Autor.

Se $z=f(x, y)$ determina uma superfície quádrica, o vetor normal a essa superfície é dado por: $\quad \vec{n}=f_{x}\left(x_{p}, y_{p}\right) \vec{\imath}+f_{y}\left(x_{p}, y_{p}\right) \vec{j} 77+\vec{k}$. qual $f_{x}\left(x_{p}, y_{p}\right)$ e $f_{y}\left(x_{p}, y_{p}\right)$ são as derivadas parciais de $z=f(x, y)$ em cada ponto $P(x, y, f(x, y))$.

A Figura 7 ilustra as etapas de obtenção do vetor ortonormal para um paraboloide. Na Figura 7a, é mostrado o vetor normal ao plano tangente num ponto. $\mathrm{Na}$ Figura 7b, o vetor normal com sentido para o interior da peça. Na Figura 7c, um conjunto de vetores normais para um valor fixo de $x$, ilustrando a diferença de módulo em cada ponto devido às diferentes taxas de variação da superfície.

Figura 7: Obtenção do vetor ortonormal a uma superfície quádrica.

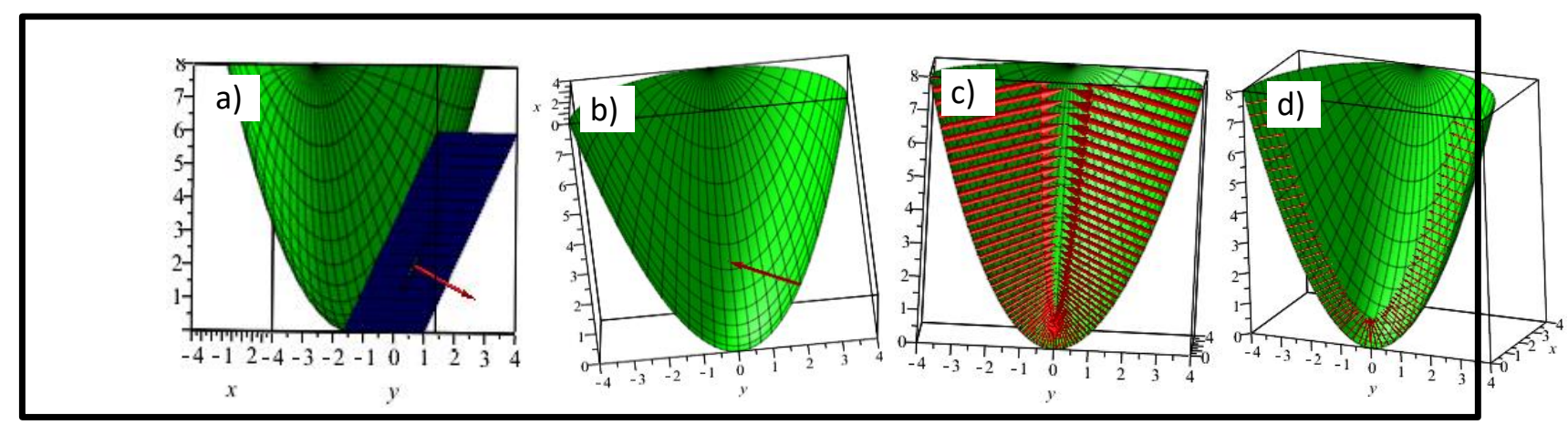

Fonte: Desenvolvido pelo Autor.

Como o vetor normal tem módulo variado, em função das diferentes taxas de inclinação da superfície em cada ponto, a solução foi utilizar o vetor ortogonal 
normalizado, ou seja, o vetor ortonormal, como ilustra a Figura 6d. Desse processo, cada ponto da extremidade da superfície, após o processo de extrusão, é dado por:

$$
P_{d}(x, y, z)=(x, y, f(x, y))-\left(f_{x}\left(x_{p}, y_{p}\right), f_{y}\left(x_{p}, y_{p}\right), 1\right) \times \lambda
$$

na qual ${ }^{\lambda}$ é a medida desejada da espessura da superfície resultante.

\section{RESULTADOS}

A aplicação da resolução dos problemas apresentados permitiu a confecção de peças com detalhes de forma e acabamento satisfatórios com relação aos objetivos iniciais. O dimensionamento e posicionamento da peça, formada pela união de cilindros, permitiu a confecção de uma peça simétrica e de bom acabamento, uma vez que cada cilindro, se colocados em posição inclinada, dispensa a utilização de material de suporte. Do mesmo modo, o processo de extrusão na direção do vetor ortonormal à superfície em cada ponto permitiu a obtenção de superfícies quádricas com espessura uniforme em todas as regiões.

A Figura 8 ilustra o processo de extrusão ortogonal à superfície. Nas Figuras $8 a$ e 8b, são mostradas duas etapas de extrusão de um Paraboloide Hiperbólico com apenas 16 subdivisões, também representando os vetores ortonormais às superfícies. Já nas Figuras 8c e 8d, é mostrada a mesma superfície, porém, com 4.096 subdivisões.

Figura 8: Processo de extrusão na direção do vetor ortonormal.

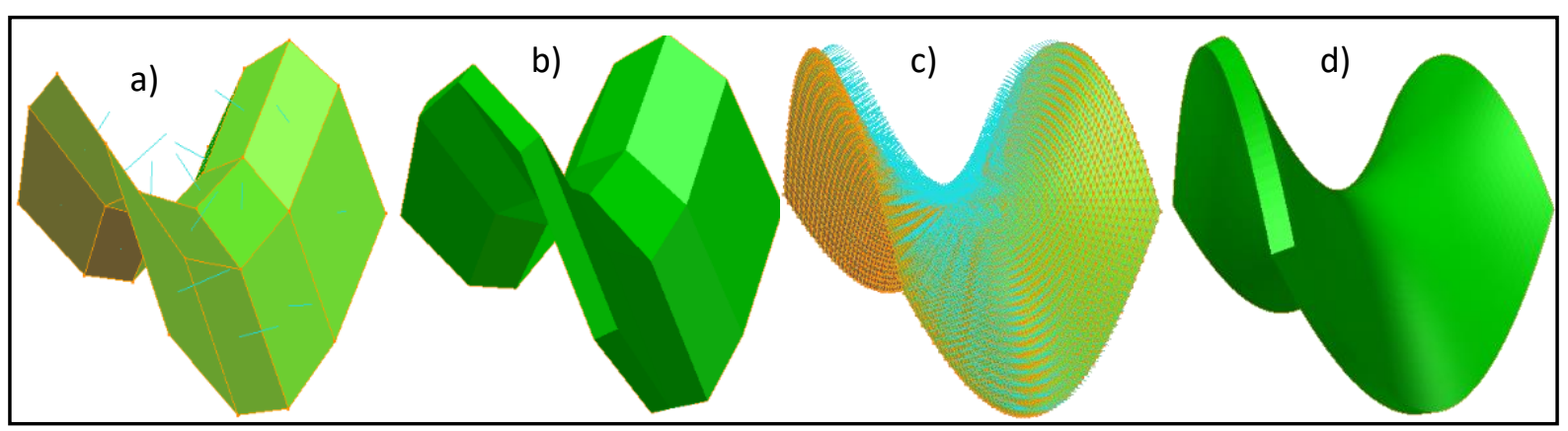

Fonte: Desenvolvido pelo Autor.

A Figura 9 mostra alguns objetos resultantes da aplicação desses resultados. Na Figura 9a, é mostrada a união de três cilindros ortogonais com um reforço esférico 
na junção. A Figura 9b mostra um Paraboloide Elíptico e a Figura 9c um Paraboloide Hiperbólico.

Figura 9: Objetos impressos utilizando as técnicas descritas.
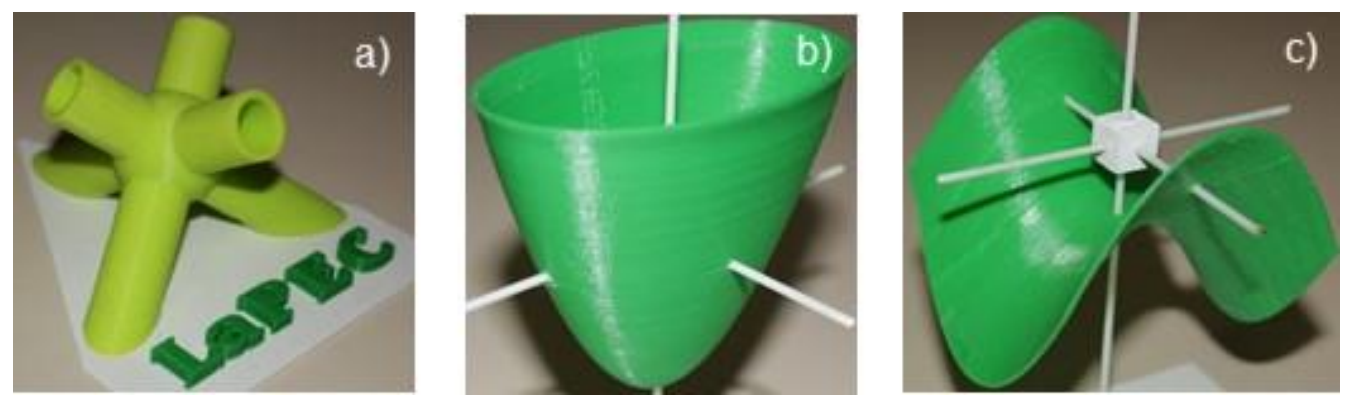

Fonte: Desenvolvido pelo Autor.

\section{CONSIDERAÇÕES}

A Pesquisa de Desenvolvimento em Projetos Experimentais, que culminou com a produção deste trabalho, permitiu a resolução de dois problemas relacionados à obtenção de formas bem definidas e de boa qualidade de impressão de objetos conceituais de matemática.

A modelagem de um sistema de três cilindros acoplados ortogonalmente, juntamente com as estratégias de cálculo de posicionamento, foi a que resultou na solução do primeiro problema e permitiu a confecção de uma peça de excelente qualidade. Já o segundo problema foi resolvido por meio da estratégia de confecção de superfícies quádricas a partir de ínfimos planos, juntamente com a obtenção da espessura uniforme das paredes por meio da extrusão na direção do vetor ortonormal. Destaca-se, nesse processo, que esse método pode ser utilizado para modelar diversas superfícies com espessura homogênea, e que pode ser aplicado em diferentes projetos de modelagem tridimensional.

As definições de estratégias de modelagem tridimensional apresentadas ancoram-se na aplicação de conceitos matemáticos, tais como a noção de limite e a extrusão na direção do vetor ortonormal à superfície em cada ponto. Desse modo, o conhecimento matemático constitui-se como base fundamental para a modelagem tridimensional precisa e de qualidade, uma vez que esta exige ampla aplicação de conceitos e conhecimentos matemáticos para a obtenção de peças bem definidas e de bom acabamento. Além disso, consolida a aprendizagem significativa dos temas e 
conceitos estudados teoricamente e enfatizados na compreensão do fenômeno sob investigação.

No que tange ao processo de aprendizado observado no desenvolvimento deste projeto experimental, denota-se a atribuição de significados, o aprofundamento e a consolidação de conceitos e conhecimentos estudados teoricamente nos cursos de Licenciatura em Física e Matemática. Em paralelo, a modelagem tridimensional, além de possibilitar a confecção de materiais didáticos inovadores, apresenta-se como uma proposta de inovação educacional, sobretudo para o ensino e a aprendizagem de ciências exatas.

\section{REFERÊNCIAS}

APPOLINÁRIO, F. Dicionário de metodologia científica: um guia para a produção do conhecimento científico. São Paulo, Atlas, 2004.

AUSUBEL, David Paul. A visão da estrutura cognitiva da palavra e do conceito de significado. In. ANDERSON, R.C. ; AUSUBEL, D. Leituras em Psicologia da Cognição. New York, Holt, Rinehart and Winston, 1965.

BARBOSA, J. C.; OLIVEIRA, A. M. P. Por que a pesquisa de desenvolvimento na Educação Matemática?. Perspectivas da Educação Matemática - UFMS - v. 8, número temático - 2015. p. 526-546.

BARROS, A. J. S.; LEHFELD, N. A. S. Fundamentos de Metodologia: Um Guia para a Iniciação Científica. 2 Ed. São Paulo, Makron Books, 2000.

COBB, P.; CONFREY, J.; DISESSA, A.; LEHRER, R.; SCHAUBLE, L. Design experiments in educational research. Educational Researcher, v. 32, n. 1, p. 9-13, jan./fev. 2003.

COLLINS, A. Toward a Design Science of Education. In: SCANLON, E.; O'SHEA, T. New directions in educational technology. Berlin, Springer Berlin Heidelberg, 1992. p. 1522.

COLLINS, A.; JOSEPH, D.; BIELACZYC, K. Design research: Theoretical and methological issues. Journal of the Learning Sciences, v. 13, n. 1, p. 15-42, 2004.

DINO, Divulgador de notícias , Impressoras 3D Impulsionam a Quarta Revolução Industrial. 2016. Disponível em: <http://exame.abril.com.br/negocios/dino/ impressoras-3d-impulsionam-a-quarta-revolucao-industrialdino89090770131. Acesso em: 03 jun. 2017.

DOERR, H. M.; WOOD, T. Pesquisa-Projeto (design research): aprendendo a ensinar Matemática. In: BORBA, M. C. (Org.). Tendências internacionais em formação de professores de matemática. Belo Horizonte, Autêntica, 2006. p. 113-128.

FONSECA, J. J. S. Metodologia da pesquisa científica. Fortaleza, UEC, 2002. Apostila. 
GARRETT, Filipe. Entenda como funcionam os diferentes tipos de impressoras 3D. Disponível em: <http://www.techtudo.com.br/listas/noticia/2016/02/entenda-comofuncionam-os-diferentes-tipos-de-impressoras-3d.html>. Acesso em: 05 ago. 2017.

PELIZZARI, A.; KRIEGL, M.L.; BARON, M.P.; FINCK, N.T.L \& DOROCINSKI, S. I. Teoria da Aprendizagem Significativa Segundo Ausubel. Revista PEC, Curitiba.,v. 2, n. 1.37-42 p. 2001/2002

SANCHEZ, Wagner. A quarta revolução industrial e seus impactos na educação. 2017?. Disponível em: <https://blog.abmes.org.br/? $p=11915$ >. Acesso em: 11 jun. 2017.

SCHWAB, K. A Quarta Revolução Industrial. Editora: EDIPRO, 2016

SZTAJN, P.; WILSON, H.; EDGINGTON, C.; MYERS, M.; DICK, L. Using Design Experiments to Conduct Research on Mathematics Professional Development. Alexandria: Revista de Educação em Ciência e Tecnologia, v. 6, n. 1, p. 9-34, abr. 2013.

TARTUCE, T. J. A. Métodos de pesquisa. Fortaleza, UNICE - Ensino Superior, 2006. Apostila.

VAZ, R. L. O uso das isometrias do Software Cabri-Gèométre como recurso no processo de prova e demonstração. 2004. 216 f. Dissertação (Mestrado em Educação), Pontifícia Universidade Católica de São Paulo, São Paulo, 2004. 